\section{Decision-making under interval uncertainty revisited}

\section{Interval uncertainty revisited}

\author{
Olga Kosheleva
}

Department of Teacher Education, The University of Texas at El Paso, El Paso, Texas, USA

Vladik Kreinovich

Department of Computer Science, University of Texas at El Paso, El Paso, Texas, USA, and

\author{
Uyen Pham
}

Faculty of Mathematical Economics, University of Economics and Law, Vietnam National University, Ho ChiMinh City, Vietnam

\begin{abstract}
Purpose - In many real-life situations, we do not know the exact values of the expected gain corresponding to different possible actions, we only have lower and upper bounds on these gains - i.e., in effect, intervals of possible gain values. The purpose of this study is to describe all possible ways to make decisions under such interval uncertainty.
\end{abstract}

Design/methodology/approach - The authors used both natural invariance and additivity requirements.

Findings - The authors demonstrated that natural requirements - invariance or additivity - led to a twoparametric family of possible decision-making strategies.

Originality/value - This is a first description of all reasonable strategies for decision-making under interval uncertainty - strategies that satisfy natural requirements of invariance or additivity.

Keywords Decision-making, Invariance, Interval uncertainty, Additivity

Paper type Research paper

\section{Formulation of the problem}

Decision-making: a brief reminder. In many real-life situations, we need to select an appropriate action. In economics, a reasonable idea is to select an action that leads to the largest values of the expected gain; e.g. Fishburn (1969), Luce and Raiffa (1989), Raiffa (1997), Nguyen et al. (2009) and Kreinovich (2014). In this manner, if we repeatedly make such a selection, then, because of the law of large numbers, we will obtain the largest possible gain.

(c) Olga Kosheleva, Vladik Kreinovich and Uyen Pham. Published in Asian Journal of Economics and Banking. Published by Emerald Publishing Limited. This article is published under the Creative Commons Attribution (CC BY 4.0) licence. Anyone may reproduce, distribute, translate and create derivative works of this article (for both commercial and non-commercial purposes), subject to full attribution to the original publication and authors. The full terms of this licence may be seen at http:// creativecommons.org/licences/by/4.0/legalcode

This work was supported in part by the National Science Foundation grants 1623190 (A Model of Change for Preparing a New Generation for Professional Practice in Computer Science) and HRD1242122 (Cyber-ShARE Center of Excellence).

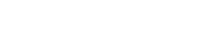


AJEB

5,1

80
Need to consider uncertainty. In practice, we often cannot predict the exact consequence of each possible action. Consequently, for each action $a$ rather than the exact value $u_{a}$ of the expected gain, we only know the interval $\left[\underline{u}_{a}, \bar{u}_{a}\right]$ of possible gain values.

Comment. It is often convenient to represent this interval in the equivalent form as $\left[\tilde{u}_{a}-\Delta_{a}, \tilde{u}_{a}+\Delta_{a}\right]$, where:

$$
\tilde{u}_{a}=\frac{\underline{u}_{a}+\bar{u}_{a}}{2} \text { and } \Delta_{a}=\frac{\bar{u}_{a}-\underline{u}_{a}}{2}
$$

How do you make decisions under interval uncertainty. How can we make decisions under such interval uncertainty? In other words, when can we decide that Action 1 is better than Action 2? In general, we have three possible cases.

Sometimes, we can guarantee that Action 1 is better than Action 2. This happens if every value $u_{1}$ from the interval $\left[\underline{u}_{1}, \bar{u}_{1}\right]$ is larger than or equal to every value $u_{2}$ from the interval $\left[\underline{u}_{2}, \bar{u}_{2}\right]$. One can easily verify that this is equivalent to requiring that the smallest possible value $\underline{u}_{1}$ from the interval $\left[\underline{u}_{1}, \bar{u}_{1}\right]$ is larger than or equal to the largest possible value $\bar{u}_{2}$ from the interval $\left[\underline{u}_{2}, \bar{u}_{2}\right]$, i.e.:

$$
\bar{u}_{1} \leq \underline{u}_{2}
$$

Sometimes, we can guarantee that Action 2 is better than Action 1, i.e., every value $u_{2}$ from the interval $\left[\underline{u}_{2}, \bar{u}_{2}\right]$ is larger than or equal to every value $u_{1}$ from the interval $\left[\underline{u}_{1}, \bar{u}_{1}\right]$. Similar to the previous case, we can conclude that this condition is equivalent to:

$$
\bar{u}_{1} \leq \bar{u}_{2}
$$

In all other cases, i.e., when

$$
\underline{u}_{2}<\bar{u}_{1} \text { and } \underline{u}_{1}<\bar{u}_{2},
$$

we cannot make a guaranteed conclusion. In such cases, Action 1 is better, and it can be that Action 2 is better.

So which action should we select? In situations in which we can guarantee that one of the actions if better, this better action is the one we should select. However, what if we are in the situation when no such guarantee is possible? Which action should we then recommend?

This is a question that we consider in this study.

Comment. It is not necessary to provide recommendation for all the cases; however, we would like to be able to provide recommendation for at least some of the cases.

\section{Analysis of the problem}

What do we want. We want to be able, for some intervals $\left[\underline{u}_{1}, \bar{u}_{1}\right]$ and $\left[\underline{u}_{2}, \bar{u}_{2}\right]$, to say that the second interval is better (or of the same quality). We will denote this relation by the usual inequality sign:

$$
\left[\underline{u}_{1}, \bar{u}_{1}\right] \leq\left[\underline{u}_{2}, \bar{u}_{2}\right]
$$

What are the natural requirements on this relation?

First natural requirement: transitivity. If Action 2 is better than (or of the same quality as) Action 1, and Action 3 is better than (or of the same quality as) Action 2, then we should be 
able to conclude that Action 3 is better than (or of the same quality as) Action 1, i.e., the relation $\leq$ on the class of all intervals should be transitive:

$$
\text { if }\left[\underline{u}_{1}, \bar{u}_{1}\right] \leq\left[\underline{u}_{2}, \bar{u}_{2}\right] \text { and }\left[\underline{u}_{2}, \bar{u}_{2}\right] \leq\left[\underline{u}_{3}, \bar{u}_{3}\right] \text {, then }\left[\underline{u}_{1}, \bar{u}_{1}\right] \leq\left[\underline{u}_{3}, \bar{u}_{3}\right] \text {. }
$$

Interval uncertainty revisited

Second natural requirement: reflexivity. Each interval has the same quality as itself. Therefore, for every interval $[\underline{u}, \bar{u}]$, we should have:

$$
[\underline{u}, \bar{u}] \leq[\underline{u}, \bar{u}] .
$$

Third natural requirement: consistency with common sense. It is reasonable to require that if Action 2 is guaranteed to be better than Action 1, then we will still select Action 2:

$$
\text { if } \bar{u}_{1} \leq \underline{u}_{2} \text {, then }\left[\underline{u}_{1}, \bar{u}_{1}\right] \leq\left[\underline{u}_{2}, \bar{u}_{2}\right] \text {. }
$$

Fourth natural requirement: scale-invariance. If we multiply all gains by the same positive constant $c>0$, then whichever gain was larger remains larger, and whichever gain was smaller remains smaller. This multiplication corresponds to switching from the original currency to the one that is $c$ times smaller: the mere change of currency should not change which action is better. Therefore, it is reasonable to require that a similar change of currency should not affect decision-making under uncertainty either, i.e.:

$$
\text { if }\left[\underline{u}_{1}, \bar{u}_{1}\right] \leq\left[\underline{u}_{2}, \bar{u}_{2}\right] \text { then }\left[c \cdot \underline{u}_{1}, c \cdot \bar{u}_{1}\right] \leq\left[c \cdot \underline{u}_{2}, c \cdot \bar{u}_{2}\right] \text {. }
$$

Fifth natural requirement: additivity. If we add the same amount to two gains, this will not change which gain is larger. Similarly, if we add the same interval-valued gain $[\underline{c}, \bar{c}]$ to the gains of both actions, this should not change which action was better.

If we have two independent situations, in one of which the gain can be anything from $\underline{u}_{i}$ to $\bar{u}_{i}$ and in the second one anything from $\underline{c}$ to $\bar{c}$, then the smallest possible value of the overall gain is when both gains are the smallest: when we have $\underline{u}_{i}+\underline{c}$ and the largest possible value of the overall gain is when both gains are the largest, i.e. when we have $\bar{u}_{i}+\bar{c}$.

Thus, the abovementioned requirement takes the following form:

$$
\left[\underline{u}_{1}, \bar{u}_{1}\right] \leq\left[\underline{u}_{2}, \bar{u}_{2}\right] \text { if and only if }\left[\underline{u}_{1}+\underline{c}, \bar{u}_{1}+\bar{c}\right] \leq\left[\underline{u}_{2}+\underline{c}, \bar{u}_{2}+\bar{c}\right] .
$$

Final natural requirement: closeness. When the values of $\underline{u}$ and $\bar{u}$ are close, the corresponding alternatives are practically indistinguishable. Thus, it is reasonable to require that if we have two sequences of intervals $\left[\underline{u}_{1}^{(n)}, \bar{u}_{1}^{(n)}\right]$ and $\left[\underline{u}_{2}^{(n)}, \bar{u}_{2}^{(n)}\right]$ for which $\left[\underline{u}_{1}^{(n)}, \bar{u}_{1}^{(n)}\right] \leq\left[\underline{u}_{2}^{(n)}, \bar{u}_{2}^{(n)}\right]$, and endpoints of both intervals tends to some limits, then because the limit intervals are indistinguishable from these one for sufficiently large $n$, we should expect the same relation $\leq$ for the limit intervals:

$$
\begin{gathered}
\text { if }\left[\underline{u}_{1}^{(n)}, \bar{u}_{1}^{(n)}\right] \leq\left[\underline{u}_{2}^{(n)}, \bar{u}_{2}^{(n)}\right] \text { for all } n \text {, and } \underline{u}_{i}^{(n)} \rightarrow \underline{u}_{i} \text { and } \bar{u}_{i}^{(n)} \rightarrow \bar{u}_{i}, \\
\text { then }\left[\underline{u}_{1}, \bar{u}_{1}\right] \leq\left[\underline{u}_{2}, \bar{u}_{2}\right] .
\end{gathered}
$$

Now, we are ready to formulate our primary result. 
AJEB

5,1

\section{Definitions and the main result} Definition

- We say that a binary relation $\leq$ on the set of all intervals is transitive if it satisfies the condition (1).

- We say that a binary relation $\leq$ on the set of all intervals is reflexive if it satisfies the condition (2).

- We say that a binary relation $\leq$ on the set of all intervals is consistent with common sense if it satisfies the condition (3).

- We say that a binary relation $\leq$ on the set of all intervals is scale-invariant if it satisfies the condition (4).

- We say that a binary relation $\leq$ on the set of all intervals is additive if it satisfies the condition (5).

- We say that a binary relation $\leq$ on the set of all intervals is closed if it satisfies the condition (6).

Proposition. For a binary relation $\leq$ on the set of all intervals, the following two conditions are equivalent to each other:

- the relation is transitive, reflexive, consistent with common sense, scale-invariant, additive and closed;

- for some values $\alpha_{-}$and $\alpha_{+}$for which $-1 \leq \alpha_{-} \leq \alpha_{+} \leq 1$, the relation $\leq$ has the following form: $\left[\tilde{u}_{1}-\Delta_{1}, \tilde{u}_{1}+\Delta_{1}\right] \leq\left[\tilde{u}_{2}-\Delta_{2}, \tilde{u}_{2}+\Delta_{2}\right]$ if and only if either:

$$
\Delta_{1} \leq \Delta_{2} \text { and } \tilde{u}_{1}+\alpha_{-} \cdot \Delta_{1} \leq \tilde{u}_{2}+\alpha_{-} \cdot \Delta_{2}
$$

or

$$
\Delta_{2} \leq \Delta_{1} \text { and } \tilde{u}_{1}+\alpha_{+} \cdot \Delta_{1} \leq \tilde{u}_{2}+\alpha_{+} \cdot \Delta_{2} .
$$

\section{Comments.}

- The only case when we have a linear order, i.e. when for every two intervals $\left[\underline{u}_{1}, \bar{u}_{1}\right]$ and $\left[\underline{u}_{2}, \bar{u}_{2}\right]$, we have either $\left[\underline{u}_{1}, \bar{u}_{1}\right] \leq\left[\underline{u}_{2}, \bar{u}_{2}\right]$ or $\left[\underline{u}_{2}, \bar{u}_{2}\right] \leq\left[\underline{u}_{1}, \bar{u}_{1}\right]$, is when $\alpha_{-}=\alpha_{+}$. In this case, we obtain the known Hurwicz criterion for decision-making, e.g., Hurwicz (1951), Luce and Raiffa (1989) and Kreinovich (2014).

- Relations described in the proposition were first considered in (Stefanini et al, 2019a) and (Stefanini et al, 2019b); however, with an additional requirement that $\left[\underline{u}_{1}, \bar{u}_{1}\right] \leq\left[\underline{u}_{2}, \bar{u}_{2}\right]$ implies $\tilde{u}_{1} \leq \tilde{u}_{2}$. This requirement is not always satisfied; see, e.g. for $\alpha_{-}=\alpha_{+}=1$, when

$$
\left[\underline{u}_{1}, \bar{u}_{1}\right] \leq\left[\underline{u}_{2}, \bar{u}_{2}\right] \text { if and only if } \bar{u}_{1} \leq \bar{u}_{2},
$$

we have $[1,1] \leq[-2,2]$. However, for the midpoints $\tilde{u}_{i}$ of these intervals, the opposite inequality is true: $1>0$.

\section{Proof}

(1) It is straightforward to confirm that every relation of the above form satisfies conditions (1)-(6). So, to complete the proof, it is sufficient to prove that if a relation satisfies the conditions (1)-(6), then it has the desired form. 
(2) Let us first analyze how the interval [-1,1] compares with different real values $u$ (i.e., with degenerate intervals $[u, u]$ ).

- Because of consistency with common sense, we have $u \leq[-1,1]$ when $u \leq-1$. Let us denote $\alpha_{-} \stackrel{\text { def }}{=} \sup \{u: u \leq[-1,1]\}$. This value is a limit of values for which $u \leq[-1,1]$; therefore, because of closeness, $\alpha_{-} \leq[-1,1]$.

By transitivity, if $u \leq \alpha_{-}$, then we have $u \leq[-1,1]$. By definition of $\alpha_{-}$, if $u>\alpha_{-}$, then we cannot have $u \leq[-1,1]$. Thus, we have:

$$
u \leq[-1,1] \text { if and only if } u \leq \alpha_{-} .
$$

- Similarly, because of consistency with common sense, we have $[-1,1] \leq u$ when $1 \leq u$. Let us denote $\alpha_{+} \stackrel{\text { def }}{=} \inf \{u:[-1,1] \leq u\}$. This value is a limit of values for which $[-1,1] \leq u$; therefore, because of closeness, $[-1,1] \leq \alpha_{+}$.

By transitivity, if $\alpha_{+} \leq u$, then we have $[-1,1] \leq u$. By definition of $\alpha_{+}$, if $u<\alpha_{+}$, then we cannot have $[-1,1] \leq u$. Thus, we have:

$$
[-1,1] \leq u \text { if and only if } \alpha_{+} \leq \mathrm{u} .
$$

(3) Let us now compare two general intervals:

$$
\left[\tilde{u}_{1}-\Delta_{1}, \tilde{u}_{1}+\Delta_{1}\right] \text { and }\left[\tilde{u}_{2}-\Delta_{2}, \tilde{u}_{2}+\Delta_{2}\right] .
$$

There are three possible cases that we will consider one by one: when $\Delta_{1}=\Delta_{2}$, when $\Delta_{1}<\Delta_{2}$, and when $\Delta_{2}<\Delta_{1}$.

- When $\Delta_{1}=\Delta_{2}$, then for $[\underline{c}, \bar{c}]=\left[-\Delta_{1}, \Delta_{1}\right]=\left[-\Delta_{2}, \Delta_{2}\right]$, additivity indicates that:

$$
\left[\tilde{u}_{1}-\Delta_{1}, \tilde{u}_{1}+\Delta_{1}\right] \leq\left[\tilde{u}_{2}-\Delta_{2}, \tilde{u}_{2}+\Delta_{2}\right] \text { if and only if } \tilde{u}_{1} \leq \tilde{u}_{2} .
$$

Therefore, in this case, the proposition is proven.

- Let us now consider the case when $\Delta_{1}<\Delta_{2}$. Then, for:

$$
[\underline{c}, \bar{c}]=\left[\tilde{u}_{2}-\Delta_{1}, \tilde{u}_{2}+\Delta_{1}\right],
$$

additivity indicates that:

$$
\begin{gathered}
{\left[\tilde{u}_{1}-\Delta_{1}, \tilde{u}_{1}+\Delta_{1}\right] \leq\left[\tilde{u}_{2}-\Delta_{2}, \tilde{u}_{2}+\Delta_{2}\right] \text { if and only if }} \\
\tilde{u}_{1}-\tilde{u}_{2} \leq\left[-\left(\Delta_{2}-\Delta_{1}\right), \Delta_{2}-\Delta_{1}\right] .
\end{gathered}
$$

By applying scale-invariance with $c=\Delta_{2}-\Delta_{1}>0$, we conclude that:

$$
\tilde{u}_{1}-\tilde{u}_{2} \leq\left[-\left(\Delta_{2}-\Delta_{1}\right), \Delta_{2}-\Delta_{1}\right] \text { if and only if } \frac{\tilde{u}_{1}-\tilde{u}_{2}}{\Delta_{2}-\Delta_{1}} \leq[-1,1] \text {. }
$$

Because of (7), this inequality is, in its turn, equivalent to:

$$
\frac{\tilde{u}_{1}-\tilde{u}_{2}}{\Delta_{2}-\Delta_{1}} \leq \alpha_{-} .
$$

Multiplying both sides of this inequality by the positive number $\Delta_{2}-\Delta_{1}$, we obtain an equivalent inequality: 
AJEB

5,1

84

$$
\tilde{u}_{1}-\tilde{u}_{2} \leq \alpha_{-} \cdot \Delta_{2}-\alpha_{-} \cdot \Delta_{1}
$$

i.e. equivalently,

$$
\tilde{u}_{1}+\alpha_{-} \cdot \Delta_{1} \leq \tilde{u}_{2}+\alpha_{-} \cdot \Delta_{2}
$$

Thus, from (9) to (13), we conclude that here, indeed:

$$
\begin{gathered}
{\left[\tilde{u}_{1}-\Delta_{1}, \tilde{u}_{1}+\Delta_{1}\right] \leq\left[\tilde{u}_{2}-\Delta_{2}, \tilde{u}_{2}+\Delta_{2}\right] \text { if and only if }} \\
\tilde{u}_{1}+\alpha_{-} \cdot \Delta_{1} \leq \tilde{u}_{2}+\alpha_{-} \cdot \Delta_{2} .
\end{gathered}
$$

- To complete the proof, we need to consider the case when $\Delta_{2}<\Delta_{1}$. Then, for $[\underline{c}, \bar{c}]=\left[\tilde{u}_{1}-\Delta_{2}, \tilde{u}_{1}+\Delta_{2}\right]$, additivity indicates that:

$$
\begin{gathered}
{\left[\tilde{u}_{1}-\Delta_{1}, \tilde{u}_{1}+\Delta_{1}\right] \leq\left[\tilde{u}_{2}-\Delta_{2}, \tilde{u}_{2}+\Delta_{2}\right] \text { if and only if }} \\
{\left[-\left(\Delta_{1}-\Delta_{2}\right), \Delta_{1}-\Delta_{2}\right] \leq \tilde{u}_{2}-\tilde{u}_{1} .}
\end{gathered}
$$

By applying scale-invariance with $c=\Delta_{1}-\Delta_{2}>0$, we conclude that:

$$
\left[-\left(\Delta_{1}-\Delta_{2}\right), \Delta_{1}-\Delta_{2}\right] \leq \tilde{u}_{2}-\tilde{u}_{1} \text { if and only if }[-1,1] \leq \frac{\tilde{u}_{2}-\tilde{u}_{1}}{\Delta_{1}-\Delta_{2}} .
$$

Because of (8), this inequality is, in its turn, equivalent to:

$$
\alpha_{+} \leq \frac{\tilde{u}_{2}-\tilde{u}_{1}}{\Delta_{1}-\Delta_{2}}
$$

Multiplying both sides of this inequality by the positive number $\Delta_{1}-\Delta_{2}$, we obtain an equivalent inequality:

$$
\alpha_{+} \cdot \Delta_{1}-\alpha_{+} \cdot \Delta_{2} \leq \tilde{u}_{2}-\tilde{u}_{1}
$$

i.e. equivalently,

$$
\tilde{u}_{1}+\alpha_{+} \cdot \Delta_{1} \leq \tilde{u}_{2}+\alpha_{+} \cdot \Delta_{2}
$$

Thus, from (15) to (19), we conclude that here indeed:

$$
\begin{gathered}
{\left[\tilde{u}_{1}-\Delta_{1}, \tilde{u}_{1}+\Delta_{1}\right] \leq\left[\tilde{u}_{2}-\Delta_{2}, \tilde{u}_{2}+\Delta_{2}\right] \text { if and only if }} \\
\tilde{u}_{1}+\alpha_{+} \cdot \Delta_{1} \leq \tilde{u}_{2}+\alpha_{+} \cdot \Delta_{2} .
\end{gathered}
$$

So, in all three cases, the proposition is confirmed. 


\section{References}

Fishburn, P.C. (1969), Utility Theory for Decision Making, John Wiley and Sons Inc., New York, NY.

Hurwicz, L. (1951), "Optimality criteria for decision making under ignorance", Cowles commission discussion paper, Statistics, Vol. 1951 No. 370.

Kreinovich, V. (2014), "Decision making under interval uncertainty (and beyond)", in Guo, P. and Pedrycz, W. (Eds), Human-Centric Decision-Making Models for Social Sciences, Springer Verlag, pp. 163-193.

Luce, R.D. and Raiffa, R. (1989), Games and Decisions: Introduction and Critical Survey, Dover, New York, NY.

Nguyen, H.T., Kosheleva, O. and Kreinovich, V. (2009), "Decision making beyond arrow's 'impossibility theorem', with the analysis of effects of collusion and mutual attraction”, International Journal of Intelligent Systems, Vol. 24 No. 1, pp. 27-47.

Raiffa, H. (1997), Decision Analysis, McGraw-Hill, Columbus, OH.

Stefanini, L., Sorini, L. and Amicizia, B. (2019a), "Interval analysis and calculus for interval-valued functions of a single variable. Part I: partial orders, gH derivative, monotonicity”, Axioms, Vol. 8 No. 4, p. 113.

Stefanini, L., Sorini, L. and Amicizia, B. (2019b), "Interval analysis and calculus for interval-valued functions of a single variable. Part II: extremal points, convexity, periodicity”, Axioms, Vol. 8 No. 4, p. 114.

\section{Corresponding author}

Vladik Kreinovich can be contacted at: vladik@utep.edu

For instructions on how to order reprints of this article, please visit our website: 